\title{
Current and future challenges in the field of powertrains
}

Prof. Dr. Jens Hadler, APL Automobil-Prüftechnik Landau GmbH

Zum Zeitpunkt der Drucklegung lagen diese Unterlagen noch nicht vor. Wir bitten um Verständnis. 